Referencia para citar este artículo: Sierra-Macías, A., Covarrubias-Bermúdez, M. de los Á., González-Pérez, G. J., \& Alfaro-Alfaro, N. (2019). Embarazos adolescentes y representaciones sociales (León, Guanajuato, México, 2016-2017). Revista Latinoamericana de Ciencias Sociales, Niñez y Juventud, 17(1), 315-325. doi:https://dx.doi. org/10.11600/1692715x.17119

\title{
Embarazos adolescentes y representaciones sociales (León, Guanajuato, México, 2016-2017)*
}

\author{
AlEJANDRa SierRa-MaCías \\ Becaria Universidad de Guadalajara, México. \\ María DE LOS ÁNGELES CoVARRUBIAS-BERMÚDEZ ${ }^{* * *}$ \\ Becaria Universidad de Guadalajara, México. \\ GUILLERMo JULIÁN GONZÁLEZ-PÉREZ **** \\ Profesor-investigador Universidad de Guadalajara, México. \\ Nó́ AlFARO-ALFARO ${ }^{* * * * *}$ \\ Profesor-investigador Universidad de Guadalajara, México.
}

\section{Artículo recibido en mayo 3 de 2018; artículo aceptado en julio 17 de 2018 (Eds.)}

- Resumen (analítico): la intencionalidad del embarazo se ha tratado de explicar desde diversos marcos teóricos, pero no siempre se considera el contexto cultural; por ello persisten complicaciones conceptuales y metodológicas. A lo largo de este estudio se pretendió identificar las representaciones sociales que las adolescentes de León (Guanajuato, México) tienen del embarazo no deseado y no planeado, estudio basado en el enfoque teórico de las representaciones sociales, con el uso de técnicas asociativas derivadas de la antropología cognitiva, específicamente listados libres y comparación de pares. Participaron 72 mujeres de 15 a 19 años. Las representaciones sociales del embarazo no planeado se centraron en aspectos económicos y emocionales negativos; el embarazo no deseado se asoció con aspectos emocionales negativos (sentimientos de enojo y culpa por la situación), además de que se consideró el aborto como solución.

Palabras claves: adolescentes, embarazo no deseado, embarazo no planeado, investigación cualitativa (Tesauro de Medical Subject Headings).

\footnotetext{
* Este artículo de investigación científica y tecnológica pertenece al área de conocimiento de la sociología, subárea temas sociales. Se basa en una investigación de diseño cualitativo «Embarazo no deseado y no planeado: las representaciones sociales de las adolescentes», realizada entre diciembre de 2016 y enero de 2017, con la financiación de las becas 227131 y 329959 otorgadas por el Consejo Nacional de Ciencia y Tecnología (Conacyt) México.

** Licenciada en Enfermería y Obstetricia de la Universidad de Guanajuato; becaria del Consejo Nacional de Ciencia y Tecnología (México) del Programa de Doctorado en Ciencias de la Salud Pública de la Universidad de Guadalajara. Orcid: 0000-0002-9685-4646. Correo electrónico: alesima120@gmail.com

*** Licenciada en Psicología de la Universidad de Guadalajara; becaria del Consejo Nacional de Ciencia y Tecnología (México) del Programa de Doctorado en Ciencias de la Salud Pública de la Universidad de Guadalajara. Orcid: 0000-0002-4419-009. Correo electrónico: angelescovarrubias@hotmail.com

**** Sociólogo y demógrafo de la Universidad de La Habana (Cuba); doctor en Ciencias de la Salud (orientación sociomédica) de la Universidad de Guadalajara (México); profesor-investigador titular de la Universidad de Guadalajara. Orcid: 0000-0003-2307-0186. Índice H5: 12. Correo electrónico: ggonzal@cencar.udg.mx

***** Médico cirujano de la Universidad Nacional Autónoma de México (Unam); doctor en Epidemiología de la Universidad de Guadalajara y de la Universidad de Washington. Profesor-investigador titular de la Universidad de Guadalajara. Orcid: 0000-0002-2827-8093. Correo electrónico: nalfaro@cucs.udg.mx
} 


\section{Alejandra Sierra-MaCías - María DE LOS ÁNGELES CoVARRUbias-BERMÚDEZ - Guillermo Julián GonZÁlEZ-PÉrEZ - NoÉ AlFARO-AlFARO}

\section{Adolescent pregnancies and social representations (León, Guanajuato, Mexico, 2016-2017)}

- Abstract (analytical): A number of authors have tried to explain the intentionality of pregnancy from different theoretical frameworks, however the cultural context is not always considered, and as a result conceptual and methodological complications persist. This study aims to identify the social representations that adolescents in León, Guanajuato construct in relation to their unwanted and unplanned pregnancies. This study is based on the theoretical approach of social representations and uses associative techniques derived from cognitive anthropology, specifically free listings and peer comparison. 72 women aged 15 to 19 participated. The social representations of unplanned pregnancy focused on negative economic and emotional aspects. Social representations of unwanted pregnancies were associated with negative emotional aspects (feelings of anger and guilt towards the situation), and abortion was considered as a solution.

Key words: adolescents, unwanted pregnancy, unplanned pregnancy, qualitative research (Thesaurus of Medical Subject Headings).

Gravidez na adolescência e representações sociais (León, Guanajuato, México, 2016-2017)

- Resumo (analítico): a intencionalidade da gravidez tem sido tratada para explicar a partir de vários referenciais teóricos, no entanto, o contexto cultural nem sempre é considerado, por isso persistem complicações conceituais e metodológicas. Este estudo tem como objetivo identificar as representações sociais que adolescentes em León, Guanajuato, têm de gravidez indesejada e não planejada. Estudo baseado na abordagem teórica das representações sociais, com o uso de técnicas associativas derivadas da antropologia cognitiva, especificamente listagens livres e comparação de pares. Participaram 72 mulheres com idades entre 15 e 19 anos. As representações sociais da gravidez não planejada focaram aspectos econômicos e emocionais negativos; a gravidez indesejada foi associada a aspectos emocionais negativos (sentimentos de raiva e culpa pela situação) e o aborto foi considerado uma solução.

Palavras-chave: Adolescentes, gravidez indesejada, gravidez não planejada, pesquisa qualitativa (Thesaurus de Medical Subject Headings).

\section{-1. Introducción. -2. Método. -3. Resultados. -4. Discusión y conclusión. -Lista de referencias.}

\section{Introducción}

La planeación del embarazo se considera un indicador de salud reproductiva, al tiempo que es clave para identificar patrones de fecundidad de las poblaciones y conocer los obstáculos de la anticoncepción (Santelli et al., 2003), demostrando así la importancia y el impacto de los programas de planificación familiar (Sedgh, Singh, \& Hussain, 2014).

Cuando se toca el tema de la intencionalidad del embarazo, generalmente se alude a embarazos no planeados o no deseados en la etapa adolescente, aunado a que se recurre a definiciones ambiguas, dejando cierta incertidumbre sobre la solidez de las medidas empleadas para indagar sobre el tema (Casas \& Solorio, 2015; Moreau, Bohet, Le Guen, Loilier, \& Bajos, 2014). Por ejemplo, se presume que cualquier comportamiento sexual es voluntario y consciente, sin considerar que está influenciado por aspectos culturales y sociales que no siempre parten de intenciones conscientes; por ende, persisten complicaciones conceptuales y metodológicas al respecto.

Varios autores mencionan que los significados que cada persona atribuye a los términos relacionados con el embarazo tienen cierta correlación con la influencia social y cultural, es decir, son construidos culturalmente (Barrett \& Wellings, 2002), por lo que difieren entre diversas poblaciones y pueden interpretarse en forma distinta (Fischer, Stanford, Jameson, \& De Witt, 1999); se ha identificado que algunas mujeres prefieren emplear el término 'inoportuno' en lugar de 'no deseado' 
a causa de las presiones sociales (Herd, Higgins, Sicinski, \& Merkurieva, 2016), por lo que debiera prestarse más atención a comprender los significados culturales y las actitudes de la gente en la toma de decisiones sobre su fecundidad (Rocca, Krishnan, Barrett, \& Wilson, 2010).

Este tema es de gran importancia en México, ya que anualmente ocurren cerca de 71 embarazos no planeados por cada 1000 mujeres en edad reproductiva (Juárez, Singh, Maddow-Zimet, \& Wulf, 2013), y se presentan con más frecuencia en áreas con mayor desarrollo socioeconómico (Menkes \& Suárez-López, 2013). De acuerdo con el Fondo de Población de las Naciones Unidas en México (UNFPA), de los embarazos en el grupo de 15 a 19 años entre el 60 y $80 \%$ son embarazos no planeados (Fondo de Población de las Naciones Unidas-UNFPA, s. f.) En el año 2009, del total de embarazos en mujeres de 15-44 años, aproximadamente el $55 \%$ no fueron planeados, y de estos el 30 $\%$ se resolvió mediante un aborto inducido (Juárez et al., 2013).

Por todo lo anterior, este tema se ha considerado un asunto tanto de salud pública como de derechos humanos que requiere mayor evidencia para identificar los significados atribuidos a los embarazos no planeados y profundizar en el tema (Menkes \& Suárez-López, 2013).

A sabiendas de que los comportamientos reproductivos están influenciados socioculturalmente, la antropología cognitiva (Bernard, 2006) y el enfoque teórico de las representaciones sociales (Abric, 2001) son una opción idónea para estudiar los saberes colectivos y de sentido común construidos de manera colectiva (Jodelet, 1984), al considerarse como un cuerpo de percepciones, creencias y opiniones respecto a un objeto, que contribuyen a la conformación y justificación de las prácticas individuales y grupales (Abric, 2001). En otros estudios se empleó este enfoque para abordar e identificar los significados construidos sobre el embarazo en adolescentes (Winkler, Pérez, \& López, 2005).

En razón de que las expectativas reproductivas están influenciadas por la cultura y la ideología, y difieren según el contexto histórico y el grupo social (Langer, \& Espinoza, 2002), surgió la inquietud de cuestionar cuáles son las representaciones sociales que las adolescentes atribuyen a los embarazos no deseados o no planeados. En el presente estudio nos planteamos como objetivo identificar las representaciones sociales que las adolescentes de León (Guanajuato) atribuyen al embarazo no deseado y al embarazo no planeado.

\section{Método}

Se realizó un estudio descriptivo y exploratorio con metodología cualitativa basada en el enfoque teórico de las representaciones sociales (Abric, 2001), durante los meses de diciembre de 2016 y enero de 2017, en la zona centro de la ciudad de León (Guanajuato), con mujeres estudiantes de secundaria y preparatoria de entre 15 y 19 años.

León está entre las primeras diez ciudades de la república mexicana con mayor número de habitantes. Su economía ha girado sobre todo hacia la producción de calzado, los servicios y la industria automotriz. Históricamente, se la ha identificado como una de las ciudades más conservadoras y tradicionalistas del país; allí se percibe un contexto político y social en el que el Estado se inclina en promover valores morales, en especial de la religión católica, entre los que sobresalen las posturas radicales antiaborto recientemente plasmadas en las constituciones locales, en las que se da cuenta de la politización de los asuntos relacionados con la salud sexual y reproductiva en la entidad, que limitan la posibilidad de decidir sobre la reproducción y el cuerpo femenino (Uribe, Rodríguez, \& Agudelo, 2015).

En total, se incluyó a 72 informantes, repartidos en 4 grupos. Dos grupos para identificar las representaciones sociales del embarazo no planeado y otros dos para conocer las relacionadas con el embarazo no deseado, en consonancia con los criterios para calcular el tamaño de muestra según Weller y Romney (1988), quienes señalan que, en estudios de descripción cultural, se requieren por lo menos 17 informantes para cumplir los criterios de suficiencia y saturación de la información. Los 


\section{Alejandra Sierra-MaCías - María DE LOS ÁNGELES CoVARRUbias-BERMÚDEZ - Guillermo Julián GonZÁlez-PÉrez - Nó́ AlFARO-AlFARO}

criterios de selección de la muestra fueron por conveniencia, buscando diversidad de las participantes por institución educativa.

Se incluyó a mujeres de entre 15 y 19 años, estudiantes de alguna institución de educación pública o privada de la zona centro de la ciudad de León. Dicha zona se caracteriza por la gran afluencia de peatones y, debido a la localización de varios recintos educativos a los alrededores, resultó fácil encontrar a mujeres estudiantes. Se las abordó mientras transitaban por la plaza de los Fundadores, en la zona centro de la ciudad; se buscó en especial a aquellas que llevaran uniforme o mochila escolar como características particulares. Se les explicó el propósito del estudio, se las invitó a participar y se solicitó su consentimiento informado. Todas las personas lo hicieron en forma voluntaria. Las entrevistas las realizó la investigadora principal, y cada participante tardó un máximo de quince minutos en responder cada cuestionario.

La recolección de los datos se llevó a cabo en dos fases sucesivas. En la primera fase del estudio se empleó la técnica de listados libres, que consistió en solicitar a 36 participantes que mencionaran las cinco primeras palabras que se les ocurrieran al leer la frase «embarazo no planeado» 0 «embarazo no deseado», y explicar posteriormente por qué eligieron cada frase (Bernard, 2006), para lo cual se les entregó una hoja por cada término inductor. A partir del análisis de los datos derivados de la aplicación de los listados libres se eligieron los diez términos más mencionados, con los cuales se diseñó el segundo instrumento, denominado comparación de pares. Este consistió en que otras 36 participantes marcaran las dos palabras que a su juicio tenían mayor relación y después las dos menos vinculadas, para luego relacionar las restantes por similitud (Abric, 2001).

El análisis de los datos de los listados libres se basó en la obtención del índice de Smith (Bernard, 2006), que es el promedio del número de veces que se menciona una palabra y el promedio del lugar en el que aparece en la lista de respuesta, para lo cual se tabularon los datos en el programa Excel $^{\mathrm{TM}}$. Para los datos del segundo instrumento, se asignó a cada par de palabras valores de 0 (menor relación) a +2 (más relación) (Abric, 2001), se ordenaron de mayor a menor y se procesaron en el programa Anthropac 4.9 (Analytic Technologies) para generar un grafo (representación gráfica de la relación de los términos utilizados para referirse al objeto social «conegma»), de acuerdo con la teoría de grafos (Doise, Clemence, \& Lorenzi-Cioldi, 2005).

Como estrategia de triangulación entre los investigadores, se hizo un análisis temático de las explicaciones relacionadas con los términos evocados en los listados libres (Minayo, 1995), que consistió en la lectura, relectura y codificación de las respuestas para categorizarlas por temas.

Finalmente, con respecto a las consideraciones éticas, se determinó que la investigación no tenía ningún riesgo para las participantes, de conformidad con el reglamento de la Ley General de Salud en Materia de Investigación para la Salud de México, publicado en el Diario Oficial de la FederaciónDOF (2014). A las participantes se les explicó el propósito del estudio, y se les aseguró tanto el anonimato como la confidencialidad de sus datos. Todas dieron su consentimiento informado de manera verbal.

\section{Resultados}

\section{Características de las informantes}

Participaron un total de 72 mujeres (de entre 15 y 19 años): 36 informantes en la fase de listados libres y otras 36 en la fase de comparación pareada; ninguna de las participantes se encontraba embarazada al momento de la encuesta y, en lo referente al estado civil, solo una de ellas estaba casada por lo civil, siendo las demás solteras; de acuerdo con la ocupación, todas eran estudiantes: 22 cursaban la secundaria y el resto eran estudiantes de preparatoria. Únicamente 11 participantes trabajaban y estudiaban. 


\section{Listados libres}

En la tabla siguiente se muestran las palabras mencionadas por las informantes respecto a los términos «embarazo no planeado» y «embarazo no deseado» (tabla 1). En ambos casos, las palabras se clasificaron en cinco categorías temáticas: facilitadores (elementos o circunstancias que contribuyen a desarrollar un embarazo no planeado o no deseado), consecuencias (aspectos negativos o positivos del embarazo), reacciones (forma de respuesta emocional que manifiesta la persona ante un embarazo), expectativas (lo que espera la persona que suceda o acontezca con el embarazo) y léxico (sinónimos de los términos).

La categoría en la que se halló mayor número de palabras evocadas por las participantes fue consecuencias, de las cuales las más mencionadas para ambos términos inductores fueron «cambiar planes de vida» y «mayor responsabilidad», en tanto que para la expresión «embarazo no deseado» la principal consecuencia fue descuidar al bebé; por otra parte, se puede apreciar una gran gama de palabras dentro de la categoría reacciones, en las que sobresalen las que aluden a estados emocionales negativos, como preocupación, tristeza, miedo y enojo.

Así mismo, se encontró que los principales facilitadores de los embarazos no planeados y no deseados se atribuyeron a factores individuales, como la falta de responsabilidad y las conductas sexuales de riesgo, al igual que a aspectos de tipo social, como la etapa de adolescencia y la información limitada; por otro lado, también se mencionaron como facilitadores los casos de violación y el consumo de alcohol, aunque en menor medida.

Respecto a las expectativas, en ambos términos las palabras se pensaron únicamente en relación con lo que sucederá con el hijo o hija, y no con la vida de la adolescente en sí; para ambos términos, la palabra más mencionada fue aborto. Por otra parte, se percibió que la población participante empleó más sinónimos para describir la expresión «embarazo no deseado», y entre los que más se mencionaron estuvieron embarazo no planeado y embarazo no querido.

Tabla 1. Palabras obtenidas en los listados libres sobre los términos embarazo no planeado y embarazo no deseado por las adolescentes en León (México), 2017

\begin{tabular}{|c|c|c|}
\hline Categorías & $\begin{array}{c}\text { Embarazo no planeado } \\
\text { Palabra (frecuencia) }\end{array}$ & $\begin{array}{c}\text { Embarazo no deseado }^{2} \\
\text { Palabra (frecuencia) }\end{array}$ \\
\hline Facilitadores & $\begin{array}{r}\text { Relaciones sexuales sin protección (18) } \\
\text { Falta de información (9) } \\
\text { Irresponsabilidad (8) } \\
\text { Calentura (excitación sexual) (5) } \\
\text { Inmadurez (4) } \\
\text { Jóvenes/adolescentes (4) } \\
\text { Falla de anticonceptivo (3) } \\
\text { Violación (2) } \\
\text { Consumo de alcohol (1) }\end{array}$ & $\begin{array}{r}\text { Irresponsabilidad (12) } \\
\text { Relaciones sexuales sin protección (6) } \\
\text { Confundir el amor con sexo (4) } \\
\text { Falta de información (5) } \\
\text { Inmadurez (4) } \\
\text { Jóvenes (3) } \\
\text { Violación (2) } \\
\text { Consumo de alcohol (1) }\end{array}$ \\
\hline Consecuencias & $\begin{array}{r}\text { Cambiar planes de vida (13) } \\
\text { Problemas económicos (10) } \\
\text { Mayor responsabilidad (9) } \\
\text { Dejar los estudios (6) } \\
\text { Descuidar al bebé (4) } \\
\text { Restringir tus necesidades (4) } \\
\text { Problemas familiares (4) } \\
\text { Conflictos con la pareja (4) } \\
\text { Riesgoso (2) }\end{array}$ & $\begin{array}{r}\text { Descuidar al bebé (13) } \\
\text { Cambiar tus planes (12) } \\
\text { Mayor responsabilidad (9) } \\
\text { Conflictos con la pareja (7) } \\
\text { Problemas económicos (7) } \\
\text { Problemas familiares (5) } \\
\text { Riesgoso (3) } \\
\text { Falta de oportunidades (1) } \\
\text { Problemas psicológicos (1) }\end{array}$ \\
\hline
\end{tabular}




\begin{tabular}{|c|c|c|}
\hline Reacciones & $\begin{array}{r}\text { Preocupación (5) } \\
\text { Miedo (4) } \\
\text { Tristeza (4) } \\
\text { Confusión (4) } \\
\text { Incertidumbre (4) } \\
\text { Enojo (3) } \\
\text { Rechazo (2) } \\
\text { Que nunca me pase a mí (2) } \\
\text { Felicidad (1) } \\
\text { Emoción (1) } \\
\text { Depresión (1) } \\
\text { Desinterés (1) } \\
\text { Decepción (1) }\end{array}$ & $\begin{array}{r}\text { Enojo (7) } \\
\text { Preocupación (6) } \\
\text { Tristeza (4) } \\
\text { Ansiedad (4) } \\
\text { Estrés (3) } \\
\text { Decepción (3) } \\
\text { Temor (3) } \\
\text { Felicidad (2) } \\
\text { Odio (2) } \\
\text { Frustración (2) } \\
\text { Coraje (1) } \\
\text { Angustia (1) } \\
\text { Depresión (1) } \\
\text { Soledad (1) } \\
\text { Confusión (1) }\end{array}$ \\
\hline Expectativas & $\begin{array}{r}\text { Aborto (3) } \\
\text { No significa que sea un error (2) } \\
\text { Dar en adopción (1) } \\
\text { Platicar y tomar decisiones (1) } \\
\text { Sacar adelante al bebé (1) }\end{array}$ & $\begin{array}{r}\text { Aborto (5) } \\
\text { Dar en adopción (2) } \\
\text { Dar un buen futuro al bebé (1) } \\
\text { No es un error (1) } \\
\text { Aceptación (1) }\end{array}$ \\
\hline Léxico & $\begin{array}{c}\text { No planeado (4) } \\
\text { No deseado (1) } \\
\text { No previsto (1) }\end{array}$ & $\begin{array}{r}\text { No planeado (4) } \\
\text { No querido (4) } \\
\text { No deseado (2) } \\
\text { Inesperado (1) } \\
\text { No buscado (1) } \\
\text { Sin intención (1) }\end{array}$ \\
\hline
\end{tabular}

Nota 1. De un total de 157 palabras.

Nota 2. De un total de 159 palabras (listados libres).

\section{Comparación de pares}

A continuación se muestra el grafo de las representaciones sociales de las mujeres participantes sobre el embarazo no planeado (figura 1). La expresión con mayor índice fue «tener más gastos», sobre la cual una de las participantes aseguró: «obviamente, con un bebé se gasta en todo», y relaciona a su vez cuatro términos, entre los que se destacan aquellos relativos a las consecuencias (tener que trabajar); al respecto, una mujer dijo: «tendría que empezar a trabajar para mantener al bebé», y en lo referente a las emociones (preocupación, miedo y tristeza), una joven mencionó: «preocupación... Cómo les diría a mis papás que estoy embarazada».

Una segunda agrupación se formó por tres términos que se relacionan con las conductas sexuales de riesgo (relaciones sexuales sin protección, «calentura» y descuido). Una de las mujeres indicó: «al no tomar las medidas anticonceptivas, es lo que obtienes». A su turno, una participante mencionó: «no le llames bendición a lo que te pasó por no usar protección», y abarcó además otros aspectos de índole religiosa. Accidente y falta de información conformaron elementos externos de la representación social. 
Figura 1. Grafo de las representaciones sociales de las adolescentes acerca del embarazo no planeado

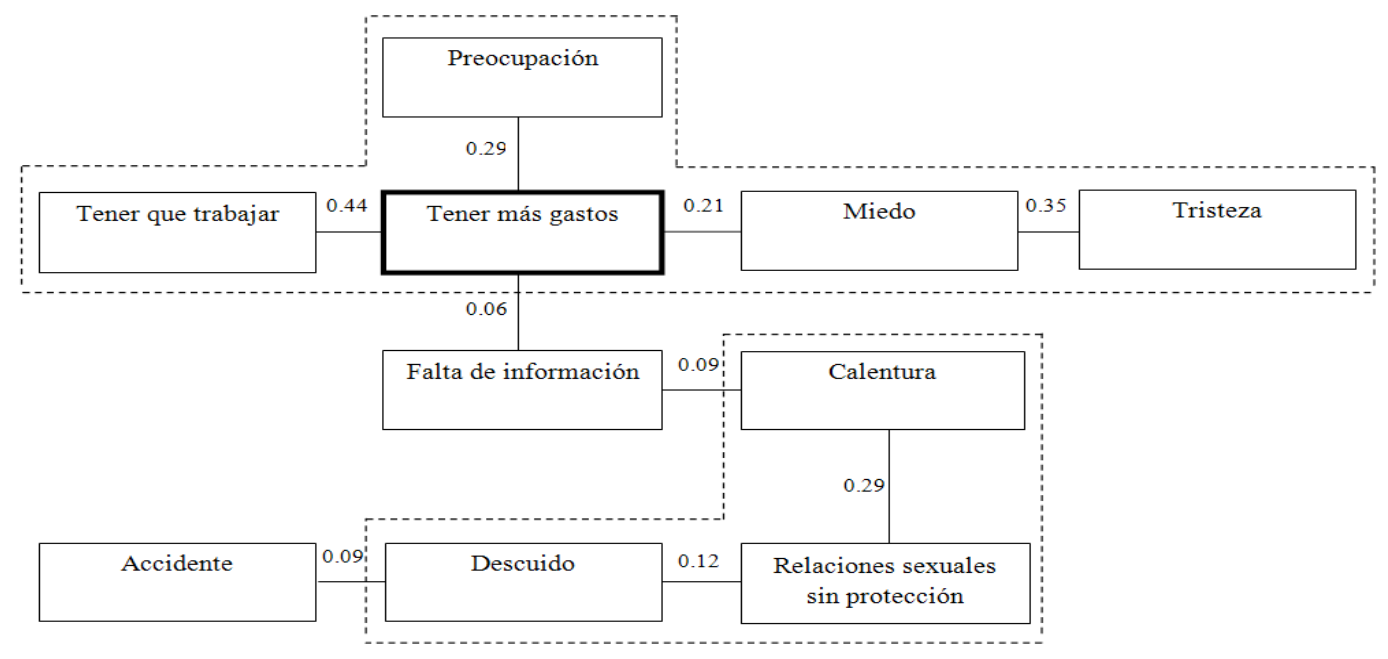

Nota. Comparación de pares embarazo no planeado. Los valores corresponden al índice de distancia.

Posteriormente, se presenta el grafo de las representaciones sociales sobre el embarazo no deseado. El término con mayor índice fue «enojo», y al respecto las participantes dijeron: «hay personas que andan de calenturientas y después no quieren.» «Te juzgan sin saber», comentarios que muestran dos puntos de vista contrapuestos; el término también se relacionó con tres conceptos (ansiedad, tristeza y estrés), y sobre esto las mujeres señalaron: «la persona no sabe qué hacer ni qué va a pasar.» «Es triste, porque los niños vienen al mundo nomás a sufrir».

Una segunda agrupación se formó con tres palabras (no planeado, cambios de planes y aborto), y respecto a esta última una de las participantes comentó: «uno busca deshacerse de lo que no quiere»; mientras otra dijo: «ahora la gente suele tener prioridades en su plan de vida y esta situación la ven como un obstáculo». Los elementos periféricos a la representación social fueron preocupación, irresponsabilidad y problemas económicos (figura 2).

Figura 2. Grafo de las representaciones sociales de las adolescentes acerca del embarazo no deseado

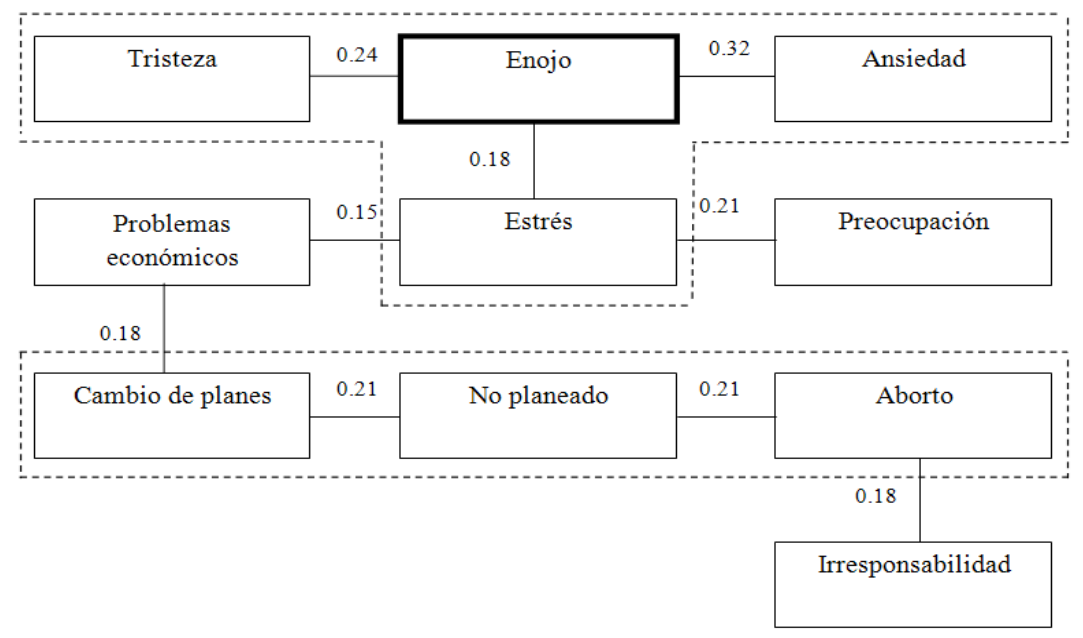

Nota. Comparación de pares embarazo no deseado. Los valores corresponden al índice de distancia. 


\section{Alejandra Sierra-MaCías - María DE LOS ÁNGELES CoVARRUbias-BERMÚDEZ - GuILLERMo Julián GoNZÁlEZ-PÉREZ - NoÉ ALFARO-ALFARO}

\section{Discusión y conclusión}

En este estudio se planteó como objetivo identificar las representaciones sociales que las adolescentes de León atribuyen al embarazo no deseado y al embarazo no planeado; de acuerdo con los resultados obtenidos en las dos fases, se muestra que para ambos términos existen algunos elementos muy similares, pero también difieren bastante en otros aspectos, por lo que cualitativamente ambos términos son distintos. En este sentido, Fischer et al. (1999) mencionan que la expresión no deseado se atribuye generalmente a la decisión de interrupción o continuidad del embarazo, en tanto que el embarazo no planificado se atribuye a la falta de uso de un método anticonceptivo (Santelli et al., 2003).

Entre los elementos más sobresalientes para ambos términos se hallaron aquellos aspectos relacionados con las consecuencias, facilitadores y reacciones, lo cual concuerda con lo que expresan otros autores, que enfatizan en que la intención de un embarazo no es solo cuestión del uso o no de anticonceptivos (Moreau et al., 2014), ya que depende de otros determinantes, como las intenciones expresadas y el deseo de la maternidad, las preparaciones preconceptuales, las influencias y las circunstancias personales de adaptación al embarazo y al bebé (Barrett, Smith, \& Wellings, 2004; Stanford, Hobbs, Jameson, De Witt, \& Fischer, 2000).

Es frecuente que cuando se habla de embarazos no planeados o no deseados, se tienda a señalar en especial a las adolescentes y los adolescentes. De acuerdo con Palma (2012), en Chile estos embarazos son más frecuentes en las mujeres jóvenes sexualmente activas. Así mismo, en Australia se reportó que los embarazos no planeados se presentan más entre las mujeres jóvenes y del Pacífico, y que son muy poco probables para las mujeres mayores de 30 años y las europeas (Hohmann-Marriott, 2018).

Si bien las relaciones sexuales sin protección y la irresponsabilidad se mencionaron como facilitadores principales, en ocasiones se considera que la iniciación o el acto sexual no siempre se producen de manera voluntaria, ya que hay casos en que suceden bajo coerción o engaño y generalmente se dan sin protección anticonceptiva. Hay que tomar en cuenta que en las sociedades latinoamericanas el hombre suele imponer su voluntad respecto al uso de anticonceptivos, aun sin recurrir a la violencia (Pantelides, 2004); ejemplo de ello son las mujeres que experimentan algún tipo de violencia, puesto que la dominación masculina limita sus posibilidades de decidir acerca de las opciones de control de la natalidad, lo cual hace más probable que ocurran embarazos no deseados (Baird, Creedy, \& Mitchell, 2017).

Cabe destacar que ambos términos inductores se relacionaron con un gran número de conceptos que abarcan aspectos emocionales negativos que repercuten en la salud mental del adolescente, sobre todo en lo relativo al momento inmediato en que se enteran del embarazo. Según Aiken y Trussell (2016), el factor más importante que subyacía en los sentimientos negativos era la falta anticipada de participación de los compañeros con el impacto emocional, físico y financiero de la maternidad no deseada; otros autores señalan que existen repercusiones posteriores al embarazo no deseado, como tener peor salud mental en la última etapa de la vida (Herd et al., 2016). En nuestro estudio se mencionó el conflicto de pareja como una consecuencia del embarazo no planeado.

Sin duda, es relevante identificar cuáles son los significados que las adolescentes atribuyen al embarazo, en relación con las expectativas; si bien el concepto de aborto se presentó para ambos casos, fue mayor para el embarazo no deseado, en concordancia con lo reportado en otros estudios, en los que se plantea que la expresión embarazo no deseado es más relevante para decidir si continuar el embarazo o interrumpirlo (Fischer et al., 1999), por lo que los primeros meses del embarazo son un momento determinante en el que las adolescentes requieren el acompañamiento y la orientación.

Por otra parte, se pudo apreciar que se mencionaron diversos sinónimos para ambos términos, pues a juicio de otros autores, el uso de categorías más claras puede contribuir a mejorar la forma en que las mujeres piensan en un embarazo, ya que los significados que cada persona atribuye a los términos relacionados con el embarazo pueden variar (Fischer et al., 1999). Tal fue el caso de un 
estudio en el que el embarazo no intencional combinó dos aspectos de la fecundidad: embarazos no deseados e inoportunos (Santelli et al., 2003). Sin embargo, de acuerdo con Moreau et al. (2014), es más probable que se hable de un embarazo como no planeado que de un embarazo no deseado.

Un aspecto que no siempre se trae a colación son las expectativas o consecuencias positivas que trae consigo el embarazo, puesto que en opinión de algunas participantes las reacciones o emociones podrían ser buenas; en tal sentido, Cater (2006) señala en su estudio que muchos de los jóvenes que fueron padres pensaron que su vida había mejorado de manera efectiva y que ahora tenían un propósito para escapar de las circunstancias familiares de pobreza, así como de aprovechar la oportunidad para corregir errores. No obstante, hoy en día hace falta mayor evidencia al respecto.

En resumen, las representaciones sociales de las adolescentes en lo referente al embarazo no planeado se centraron principalmente en aspectos económicos (tener más gastos) y en aspectos emocionales negativos, relativos a la forma o el momento en que les comunicarán a sus familiares sobre el embarazo, en tanto que el embarazo no deseado se relacionó con sentimientos de enojo, culpa e impotencia a causa de la situación y su significado (¿qué voy a hacer ahora?), al tiempo que se plantearon soluciones como el aborto y el abandono de metas personales. Esto concuerda con un estudio llevado a cabo con adolescentes de Ghana, en el que se exploraron las experiencias y percepciones del embarazo no deseado (Aziato et al., 2016).

Por medio de este estudio se buscó contribuir a identificar algunas de las representaciones sociales de la población en torno a las intenciones de fecundidad, a partir del conocimiento de sentido común de los propios actores, mediante estrategias de recolección de información sencillas y rápidas, que facilitaran la transferibilidad metodológica y que los resultados propiciaran el diseño o rediseño de programas de salud pública que comprendan los significados culturales y actitudes de las personas en la toma de decisiones de fecundidad, que tienen que ver directamente con los derechos sexuales y reproductivos, eficaces para prevenir embarazos no planeados y no deseados.

Por último, la principal limitación de este estudio fue el énfasis en los aspectos cognitivos de la representación social, ya que no se consideran los procesos participativos, las prácticas y los niveles de análisis grupal. Para futuros estudios se sugiere incluir las subjetividades de los propios adolescentes, así como también la mirada de los hombres adolescentes y de otros tipos de población, estratificando por grupos de edad y en diferentes condiciones sociales.

\section{Lista de referencias}

Abric, J. C. (2001). Prácticas y representaciones sociales. Ciudad de México: Ediciones Coyoacán. Aiken, A. R., \& Trussell, J. (2016). Anticipated emotions about unintended pregnancy in relationship context: Are latinas really happier? Journal of Marriage and Family, 79(2), 356-371. doi:10.1111/ jomf.12338.

Aziato, L., Hindin, M. J., Maya, E. T., Manu, A., Amuasi, S. A., Lawerh, R. M., \& Ankomah, A. (2016). Adolescents' responses to an unintended pregnancy in Ghana: A qualitative study. Journal of Pediatric and Adolescent Gynecology, 29(6), 653-658. doi:10.1016/j.jpag.2016.06.005.

Baird, K., Creedy, D., \& Mitchell, T. (2017). Intimate partner violence and pregnancy intentions: A qualitative study. Journal of Clinical Nursing, 26(15-16), 2399-2408. doi:10.1111/jocn.13394.

Barrett, G., Smith, S. C., \& Wellings, K. (2004). Conceptualisation, development, and evaluation of a measure of unplanned pregnancy. Journal of Epidemiology and Community Health, 58(5), 426433. doi:10.1136/jech.2003.014787.

Barrett, G., \& Wellings, K. (2002). What is a «planned» pregnancy? Empirical data from a British study. Social Science \& Medicine, 55(4), 545-557. doi:10.1016/S0277-9536(01)00187-3.

Bernard, H. R. (2006). Métodos de investigación en antropología: abordajes cualitativos y cuantitativos. Walnut Creek, Cal.: Altamira Press. 


\section{Alejandra Sierra-MaCías - María DE LOS ÁNGELES CoVARRUbias-BERMÚDEZ - Guillermo Julián GonZÁlez-PéreZ - Nó́ AlFARO-ALFARO}

Casas, J., \& Solorio, C. (2015). Jóvenes en edad escolar y embarazo no planeado. Reflexiones, 94(2), 79-87.

Cater, S., \& Coleman, L. (2006). «Planned» teenage pregnancy: Perspectives of young parents from disadvantaged backgrounds. Bristol: The Policy Press.

Diario Oficial de la Federación-DOF. (2014). Decreto por el que se reforman, adicionan y derogan diversas disposiciones del Reglamento de la Ley General de Salud en Materia de Investigación para la Salud. Recuperado de: http://www.dof.gob.mx.

Doise, W., Clemence, A., \& Lorenzi-Cioldi, F. (2005). Representaciones sociales y análisis de los datos. Ciudad de México: Instituto Mora.

Fischer, R. C., Stanford, J. B., Jameson, P., \& De Witt, M. J. (1999). Exploring the concepts of intended, planned, and wanted pregnancy. Journal of Family Practice, 48, 117-122.

Fondo de Población de las Naciones Unidas (UNFPA). (s. f.) Salud sexual y reproductiva en adolescentes y jóvenes. Recuperado de: http://mexico.unfpa.org/es/topics/salud-sexual-yreproductiva-en-adolescentes-y-j\%C3\%B3venes.

Herd, P., Higgins, J., Sicinski, K., \& Merkurieva, I. (2016). The implications of unintended pregnancies for mental health in later life. American Journal of Public Health, 106(3), 421-429. doi:10.2105/AJPH.2015.302973.

Hohmann-Marriott, B. E. (2018). Unplanned pregnancies in New Zealand. Australian and New Zealand Journal of Obstetrics and Gynaecology, 58(2), 247-250. doi:10.1111/ajo.12732.

Jodelet, D. (1984). La representación social: fenómenos, concepto y teoría. En S. Moscovici (Ed.), Psicología Social II (pp. 469-494). Barcelona: Paidós.

Juárez, F., Singh, S., Maddow-Zimet, I., \& Wulf, D. (2013). Embarazo no planeado y aborto inducido en México: causas y consecuencias. Nueva York: Guttmacher Institute.

Langer, A., \& Espinoza, H. (2002). El embarazo no deseado: impacto sobre la salud y la sociedad en América Latina y el Caribe. Pan American Journal of Public Health, 11, 192-204.

Menkes, C., \& Suárez-López, L. (2013). El embarazo de los adolescentes en México: ¿es deseado. Coyuntura Demográfica, 4, 21-28.

Minayo, C. (1995). El desafio del conocimiento: investigación cualitativa en salud. Buenos Aires: Lugar.

Moreau, C., Bohet, A., Le Guen, M., Loilier, A. R., \& Bajos, N. (2014). Unplanned or unwanted? A randomized study of national estimates of pregnancy intentions. Fertility and Sterility, 102(6), 1663-1670. https://doi.org/10.1016/j.fertnstert.2014.08.011.

Palma, I. (2012). Las nuevas generaciones de mujeres, el embarazo no previsto, las edades y la segmentación social en la sociedad chilena. Revista Médica de Chile, 140(3), 319-325. doi:10.4067/S0034-98872012000300006.

Pantelides, E. A. (2004). Aspectos sociales del embarazo y la fecundidad adolescente en América. Notas de Población, 78, 7-34.

Cámara de Diputados del H. Congreso de la Unión. (1987). Reglamento de la Ley General de Salud en Materia de Investigación (1987). Última reforma publicada. DOF 02-04-2014. Recuperado de: http://www.diputados.gob.mx/LeyesBiblio/regley/Reg_LGS_MIS.pdf

Rocca, C. H., Krishnan, S., Barrett, G., \& Wilson, M. (2010). Measuring pregnancy planning: An assessment of the London Measure of Unplanned Pregnancy among urban, south Indian women. Demographic Research, 23, 293-334.

Santelli, J., Rochat, R., Hatfield-Timajchy, K., Gilbert, B. C., Curtis, K., Cabral, R., \& Schieve, L. (2003). The measurement and meaning of unintended pregnancy. Perspectives on Sexual and Reproductive Health, 35(2), 94-101. doi:10.1363/3509403.

Sedgh, G., Singh, S., \& Hussain, R. (2014). Intended and unintended pregnancies worldwide in 2012 and recent trends. Studies in Family Planning, 45(3), 301-314. doi:10.1111/j.17284465.2014.00393.x. 
Stanford, J. B., Hobbs, R., Jameson, P., De Witt, M. J., \& Fischer, R. C. (2000). Defining dimensions of pregnancy intendedness. Maternal and Child Health Journal, 4(3), 183-189. doi:10.1023/A:1009575514205.

Uribe, M., Rodríguez, K., \& Agudelo, M. (2015). Salud sexual y reproductiva en México: determinantes sociales y acceso a los servicios del Seguro Popular en el municipio de León (Guanajuato). Buenos Aires: Clacso.

Weller, S., \& Romney, K. (1988). Systematic data collection. Londres: Sage.

Winkler, M. I., Pérez, C. P., \& López, L. (2005). ¿Embarazo deseado o no deseado?: representaciones sociales del embarazo adolescente, en adolescentes hombres y mujeres, habitantes de la comuna de Talagante, Región Metropolitana. Terapia Psicológica, 23(2), 19-31. 